\title{
Equipamiento para el Aprendizaje de los Fundamentos de Transferencia de Cantidad de Movimiento, de Energía y de Materia
}

\author{
E.D. Albizzati, A.N. Arese, D.A. Estenoz y G.H. Rossetti \\ Univ. Nacional del Litoral, Facultad de Ing. Química, Departamento de Ingeniería Química, \\ Santiago del Estero 2654, (3000) Santa Fe-Argentina (e-mail: arese@fiqus.unl.edu.ar)
}

\begin{abstract}
Resumen
En este trabajo se presenta una experiencia, sobre la realización de trabajos prácticos grupales, con participación activa de los alumnos en cursos de ingeniería. Las actividades que se describen están destinadas al diseño, construcción y ensayo de un equipo didáctico para la medición de propiedades de transporte tales como, coeficientes de transferencia de cantidad de movimiento, energía y materia. La realización del trabajo práctico persigue objetivos que van más allá del aprendizaje de ciertos conceptos, pues se pretende que los alumnos adquieran capacidades, aptitudes y actitudes no contempladas en los métodos tradicionales de enseñanza. La metodología aplicada, que ha generado gran interés en los estudiantes, favorece la formación en el campo de las relaciones interpersonales, brinda oportunidades para la comunicación oral y escrita y contribuye a una mejor comprensión de conceptos por parte del alumno. Sin embargo, el método requiere de una mayor dedicación y esfuerzo de los docentes.
\end{abstract}

\section{Equipment for the Learning of Momentum, Heat and Mass Transfer Fundamentals}

\begin{abstract}
This paper presents an experience about groupal practical works with active participation of students in chemical engineering courses. The activities being described aim to the design, construction and testing of didactic equipment to measure transport properties such as momentum, energy and mass transfer coefficients. This practical work, going beyond the learning of certain concepts, intends that students acquire capacities, skills and attitudes not considered in traditional teaching methods. The applied methodology, which has generated great interest among students, favors the training in the field of interpersonal relationships, offers opportunities for oral and written communication and contributes to better students understanding. However. the method requires more dedication and effort by instructors.
\end{abstract}

Keywords: heat transfer, engineering education, didactic equipment, groupal work

Nota: este artículo está tomado de "Información Tecnológica" [ISSN 0716-8756], vol. 9(4), 349-355 (1998) 


\section{INTRODUCCIÓN}

El desempeño profesional del Ingeniero Químico raras veces implica la realización de tareas según una secuencia rígida preestablecida, lo que en general suele caracterizar a las experiencias de laboratorio de gran parte de las asignaturas de la carrera, principalmente aquéllas de los primeros años. Por ello, en la mayoría de los casos, los estudiantes no tienen oportunidad de apreciar ciertos aspectos de la práctica ingenienil hasta la fase (mal de la carrera (Scarrah, 1979). Asimismo, los rápidos cambios de la tecnología generalmente no producen los cambios correspondientes en la educación de los ingenieros, y en buena parte, la enseñanza de la ingeniería conserva los métodos tradicionales. En los cursos se acostumbra a dar demasiado énfasis a los problemas cerrados y claramente enunciados, y muy poco énfasis a la formulación de problemas y a los problemas abiertos en los cuales puede haber más de una respuesta correcta (Ray Bowen,1994).

Es sabido que para lograr un aprendizaje significativo, no memorístico de las Ciencias se requiere el dominio del método con que se han construido y se construyen los conocimientos asociados a las mismas (Concari el al., 1992). El conocimiento científico se construye a partir de una idealización, abstracción y modelado de la realidad. El modelado no sólo es una estrategia cognoscitiva empleada en las ciencias "puras", sino también una herramienta fundamental en la actividad tecnológica y en el diseño ingenieril (Salinas de Sandoval et al., 1995a). Por este motivo, el alumno debe incorporar el pensamiento científico de modo que pueda usarlo a lo largo de la carrera y en su vida profesional, para explicar, modelar y transformar el medio ambiente que lo rodea.

Por otra parte, las situaciones problemáticas y la interpretación y explicación de los fenómenos, son los ejes vertebradores de la construcción científica, como así también de la práctica profesional de los Ingenieros. Si se pretende aproximar la labor de los estudiantes a una práctica científica, se deberían "planificar actividades guiadas que enfrenten colectivamente a los estudiantes con situaciones problemáticas abiertas, motivadoras y accesibles, que ya hayan sido estudiadas y respondidas en alguna medida por la comunidad científica, y de las que el docente tenga un adecuado manejo" (Salinas de Sandoval et al., 1995b).

Este trabajo se refiere a una experiencia llevada a cabo en la Cátedra de Fundamentos de la Ingeniería Química, relacionada con Trabajos Prácticos grupales. A partir de 1989 se procedió a revisar y modificar la metodología de enseñanza y aprendizaje, y las mayores innovaciones se realizaron en el Trabajo Práctico y en la evaluación de la asignatura (Albizzati y Arese, 1994). Las actividades desarrolladas durante el Trabajo Práctico están destinadas al diseño, construcción y ensayo de un equipo didáctico, y se encuadran en lo que se conoce como "método de proyectos" (Nérici, 1980). Los equipos que aquí se describen están orientados al análisis fenomenológico y conceptual, y a la medición de propiedades de transporte y coeficientes de Transferencia de Cantidad de Movimiento, de Energía y de Materia. La realización del Trabajo Práctico persigue objetivos que van más allá del aprendizaje de contenidos ya que se pretende que el alumno adquiera capacidades, aptitudes y actitudes no siempre contempladas a través de otros métodos de enseñanza y aprendizaje: se estimula el pensamiento creativo, el análisis critico, el razonamiento y la iniciativa personal de los estudiantes, entre otros. Los referidos aspectos no necesariamente están todos involucrados en las metodologías tradicionales aplicadas en los trabajos prácticos de las carreras de Ingeniería.

\section{CARACTERÍSTICAS DEL TRABAJO PRÁCTICO}

Con respecto al Trabajo Práctico, tiene características extra-áulicas y grupal, efectuándose con la participación activa de los estudiantes. Consiste globalmente en el planeamiento, ejecución y comunicación escrita y oral de un proyecto, relacionado con el diseño, construcción y ensayo de un equipo didáctico destinado al estudio de uno de los temas de la asignatura. Durante la realización del Trabajo Práctico, a cada grupo se le asigna un docente asesor quien, a requerimiento de los integrantes, los orienta durante la preparación y ejecución del trabajo, y supervisa el desarrollo de las actividades teniendo en cuenta los objetivos que se persiguen. 
En el Trabajo Práctico, los estudiantes desarrollan un tema elegido por ellos mismos a partir de las propuestas efectuadas por la cátedra y en el marco de los contenidos del programa. En función de la complejidad del tema, tiempo estimado de concreción y particularidades de cada trabajo, el número de estudiantes por comisión varía entre dos y tres. La integración de los grupos surge de los propios alumnos. Las actividades se programan de forma tal que cada tema es abordado por más de un grupo, en progresivas etapas. Las tareas se encadenan y los resultados obtenidos por un grupo en un determinado período son revisados en el período siguiente por otro grupo el cual luego planifica y ejecuta sus propias actividades. De este modo se completa el estudio de un tema, con el aporte sucesivo de varias comisiones. En general los Trabajos Prácticos se desarrollan según las etapas que se listan en la Tabla 1.

Tabla 1: Desarrollo del Trabajo Práctico

\begin{tabular}{|l|}
\hline \multicolumn{1}{|c|}{ Etapas } \\
\hline - análisis teórico \\
- diseño y construcción de equipos \\
- modelado y/o simulación \\
- obtención de datos experimentales \\
- interpretación de resultados \\
- elaboración de conclusiones \\
- mejora de detalles constructivos y de técnicas de medición \\
- presentación oral y escrita \\
\end{tabular}

En todos los casos, en principio se contemplan aspectos teóricos, se profundizan conocimientos impartidos previamente en la asignatura, se realizan conceptualizaciones y estudios analíticos a partir de artículos publicados en libros y revistas técnico-científicas, los que se recopilan mediante búsquedas bibliográficas. Luego se efectúa un análisis comparativo de la información relacionada con los métodos experimentales disponibles y, considerando los recursos técnicos y materiales, así como las posibilidades de concreción en plazos razonables, se selecciona el dispositivo de acuerdo a los objetivos generales planteados.

La etapa de modelado y/o simulación del sistema en estudio se realiza según las necesidades, previamente al diseño, en algunos casos, o con posterioridad al mismo, en otros. De este modo se predice el funcionamiento de los equipos para contribuir a la selección del instrumental de medición, elaboración de técnicas operatorias y dimensionamiento de instalaciones complementarias. En lo referente a las determinaciones experimentales, las mismas se concretan en los equipos previamente desarrollados. En un tema donde la etapa experimental obliga a contar con equipamiento de características particulares y de cierta complejidad, se usó un equipo comercial.

Los datos de las experiencias se contrastan con información obtenida por otras vías y se interpretan con el fin de extraer conclusiones sobra los modelos teóricos aplicados, para proceder a su reformulación en caso necesario. Además se efectúa un análisis crítico de las tareas realizadas, lo que habitualmente da lugar a la corrección de detalles constructivos y al perfeccionamiento de las técnicas de medición utilizadas en cada caso. Una vez finalizado el Trabajo Práctico, los alumnos presentan un informe escrito y exponen oralmente los resultados del trabajo al resto de los estudiantes y a los docentes, durante aproximadamente treinta minutos cada grupo, incluyéndose un periodo de discusión conjunta del tema. 


\section{EQUIPAMIENTO DIDÁCTICO}

A continuación se refiere a aquellos aspectos más significativos relacionados con los equipos didácticos. En la Tabla 2 se listan los temas desarrollados en las áreas de Transferencia de Cantidad de Movimiento, de Energía y de Materia.

Tabla 2: Equipos desarrollados

Transferencia de Cantidad de Movimiento
- Predicción y visualización de flujos
- Caracterización de fluidos no newtonianos
- Perfiles de velocidad en fluidos newtonianos
- Movimiento de gotas en medios fluidos
- Descarga de líquidos desde tanques
- Flujo en cañerías, accesorios y válvulas
Transferencia de Energía
- Conductividad térmica de un aislante
- Perfiles de temperatura en barras metálicas
- Calentamiento de un liquido en tanque agitado
- Colector solar plano para calentamiento de agua
- Transferencia de calor desde superficies planas
- Intercambiador de calor de tubos concéntricos
Transferencia de Materia
- Difusividad de vapores en aire
- Perfiles de concentración de sal en queso
- Transferencia de materia desde gotas a líquidos
- Disolución de sólidos en líquidos agitados
- Aireación de líquidos residuales
- Evaporación desde una película descendente

Se ha diseñado y construido un primer grupo de equipos teniendo como base los esquemas y detalles de la bibliografía, que incluye experiencias "típicas" para el estudio de estos temas, pero modificando varias de las propuestas en lo relativo a objetivos, modelos teóricos, elementos constructivos e instrumental de medición, alcanzando así una modalidad propia en la aplicación de cada uno de estos equipos didácticos. En función de las características de la información bibliográfica disponible, se procedió al diseño y construcción de un segundo grupo de equipos a partir de una elaboración propia de los estudiantes, y a la vez, a la planificación del modo de uso de los equipos didácticos desarrollados. Aunque las pautas seguidas en estos dispositivos fueron análogas a las aplicadas en la adaptación de los equipos clásicos referidos con anterioridad, puede decirse que en general el avance fue más lento. En todos los casos esto dio lugar a un análisis más crítico, surgieron discusiones en lo que respecta a detalles constructivos, modelado teórico, técnicas de medición e interpretación de resultados experimentales. Fue necesario contrastar la información obtenida con la proveniente de revistas y libros. Así se reformularon los modelos y se redefinieron los objetivos del Trabajo Práctico en función de las primeras conclusiones elaboradas. Seguidamente se describen brevemente los equipos, considerando los grupos (I y II) anteriormente mencionados.

\section{Grupo I: equipos clásicos con experiencias típicas}

Se realizaron experiencias en equipos clásicos y disponibles para la predicción y visualizaron de flujos, equipos para la medición de conductividad térmica de un aislante y se ha medido la difusividad de distintos vapores orgánicos en aire. Estos experimentos corresponden a materias de transferencia de cantidad de movimiento, transferencia de energía y transferencia de materia, como se detalla en lo que sigue. 


\section{a) Transferencia de Cantidad de Movimiento}

Se realizó una experiencia para la predicción y visualizaron de flujos (Giorgetti, 1985). Fue desarrollado un equipo para observar las líneas de comente alrededor de objetos sumergidos en un fluido que se mueve con flujo laminar y estacionario; se ha experimentado con agua y mezclas de glicerina-agua, utilizándose diferentes trazadores (partículas finamente divididas, colorantes, etc.). Los perfiles obtenidos experimentalmente se compararon con las gráficas resultantes de la solución de un modelo teórico que representa el flujo alrededor de objetos de diferentes formas geométricas. También, se construyó un equipo para medir perfiles de velocidad en fluidos newtonianos y otro para determinar las pérdidas por fricción en cañerías, accesorios y válvulas (Crosby, 1968). Se determinaron perfiles de velocidad en agua circulando por una cañería con régimen laminar y turbulento. Dichos perfiles se confrontaron con los obtenidos teóricamente a partir de la ecuación local de balance de cantidad de movimiento (régimen laminar) y a través de ecuaciones semiempíricas (régimen turbulento).

En el equipo construido para la determinación de pérdidas de energía, se calculó a través de la medición de la presión y el caudal de agua circulante, el coeficiente de fricción de Darcy para distintos diámetros de cañería y diferentes materiales, observándose el efecto de la rugosidad. Con los mismos datos experimentales y a través de un balance de energía mecánica se determinaron los coeficientes de pérdidas en válvulas y accesorios. Se estudió la descarga de un líquido desde un tanque (Crosby, 1968) por un orificio ubicado en el fondo del mismo, midiéndose la variación de la altura del líquido dentro del tanque mientras éste se vacía, con y sin tubo de descarga. Con los datos experimentales y un balance de energía mecánica se calculó el coeficiente de pérdidas de energía en el orificio. Otras experiencias se realizaron operando el dispositivo anterior en estado estacionario para determinar las pérdidas por fricción en función de la longitud de tubo. Con el equipo puede compararse el comportamiento de un fluido simple como el agua con fluidos no newtonianos (por ejemplo soluciones acuosas de carboximetil-celulosa al 1.5\%).

\section{b) Transferencia de Energía}

Se han desarrollado equipos para medición de conductividad térmica de un aislante según la propuesta de Molyneux (1969) y para la determinación de perfiles de temperatura en barras sólidas (Crosby, 1968). En el primer caso se ha incluido el estudio de la variación de la conductividad térmica del aislante con la temperatura, y en el segundo el efecto de la temperatura sobre el valor del coeficiente local de transferencia de energía desde la superficie de la barra al ambiente. Además se estudió la incidencia del flujo de aire en convección forzada y natural, sobre la transferencia de calor.

Se llevaron a cabo experiencias relativas al cálculo y la verificación experimental de coeficientes totales e individuales de transferencia de calor en un tanque agitado con serpentín interno y en un intercambiador de tubos concéntricos (Crosby, 1968). En ambos equipos el calentamiento se efectuó utilizando vapor de agua a baja presión. En el tanque agitado se investigó la variación del coeficiente total de transferencia de energía con el tiempo y con la velocidad del agitador. En los trabajos prácticos empleando el intercambiador de tubos concéntricos, se determinó el efecto del caudal másico de fluido calentado sobre el coeficiente total y el coeficiente convectivo interno de transferencia de energía usando el método de Wilson (Greenkom y Kessler, 1972).

\section{c) Transferencia de Materia}

Se ha medido la difusividad de distintos vapores orgánicos en aire haciendo uso de un dispositivo termostatizado (Molyneux, 1969). Se analizó la influencia de la temperatura sobre el valor de la propiedad para verificar las expresiones usadas para predicción de la difusividad (Reid et al., 1987). Asimismo se ha determinado el valor del coeficiente convectivo de transferencia de materia en la fase gaseosa empleando una columna con película de agua descendente. Para medir la humedad y temperatura de la corriente de aire a la salida del equipo se utilizó un termo higrómetro portátil, hallándose en las experiencias la variación del coeficiente de transferencia con el caudal de aire circulante (Molyneux, 1969). Se efectuó paralelamente un estudio relacionado con la formación de la película descendente y el efecto del caudal circulante sobre dicha conformación. 


\section{Grupo II: propuestas de equipos y experiencias}

Al igual que en el caso anterior, en este grupo se realizaron experimentos relacionados con transferencia de cantidad de movimiento, de energía y de materia en equipos nuevos. Se construyó un dispositivo para estudiar el movimiento de gotas en el seno de un fluido, se estudió la transferencia de energía desde una superficie plana y se estudiaron los perfiles de concentración de cloruro de sodio en la etapa de maduración de quesos.

\section{a) Transferencia de Cantidad de Movimiento}

Se construyó un dispositivo para estudiar el movimiento de gotas en el seno de un fluido. El equipo consta de una columna de vidrio que contiene al medio fluido dentro del cual descienden las gotas inmiscibles. En las experiencias se determina el coeficiente de fricción de una partícula esférica fluida (de agua) que se mueve en el seno de un fluido inmiscible (vaselina), usando para el cálculo la fuerza de fricción obtenida mediante un balance de fuerzas aplicado a la gota. Estos resultados se contrastaron con los que se obtienen aplicando la teoría de Hadamard-Rybczynsky (Papanastasiou, 1994), comprobándose diferentes comportamientos al utilizar agua de red o desmineralizada.

\section{b) Transferencia de Energía}

Se estudió la Transferencia de Energía desde una superficie plana (Incropera y De Witt, 1990). Para efectuar el estudio se adoptó la forma de placa circular, analizándose el efecto del tipo de superficie sobre el valor del coeficiente de transferencia de energía y del flujo de calor transferido por convección natural y por radiación térmica. Las experiencias se llevaron a cabo con placas de acero inoxidable cubierta con una pintura negra mate, en un caso, y muy pulida en el otro. Los resultados experimentales sirvieron para discutir sobre las restricciones de las correlaciones propuestas en la literatura. En relación al calentamiento de agua utilizando energía solar se construyeron colectores solares tipo compacto (Garg y Rani, 1982) con una placa negra absorbente de la radiación solar. Uno de los colectores con la cubierta transparente la radiación de vidrio común y el restante de poli carbonato alveolar, ambas con el mismo espesor. Se realizaron determinaciones de la eficiencia térmica de los equipos operados en forma discontinua, bajo diversas condiciones de irradiación solar y en diversas épocas del arto, concluyéndose sobre las condiciones que redundan en un mayor rendimiento de cada uno de los colectores de placa plana construidos.

\section{c) Transferencia de Materia}

Se estudiaron los perfiles de concentración de cloruro de sodio en la etapa de maduración de quesos de pasta semidura. Para ello se elaboraron quesos tipo Pategras, hallándose la variación de la concentración salina con el tiempo y con la posición radial en quesos cilíndricos cuyas caras inferior y superior fueron recubiertas con el objeto de permitir la penetración de sal sólo por la superficie lateral. El modelo teórico que describe el sistema fue verificado a través de los resultados experimentales (Meinardy et al., 1995).

Otros equipos didácticos se destinaron al análisis de los fenómenos de transferencia de materia entre dos fases (líquido-líquido, sólido-líquido y gas-líquido). Los dispositivos se usaron para estudiar el flujo de ácido propiónico desde gotas de benceno hacia agua, disolución de esferas de naftaleno en soluciones acuosas de etanol y aireación en tanques agitados, de suero residual de la fabricación de quesos. Uno de los dispositivos básicamente está constituido por una columna donde se forman y ascienden gotas de benceno que contienen ácido propiónico. El ácido se transfiere desde la gota a la fase continua, que es agua (Camacho Rubio et al., 1981).

Se halló el coeficiente promedio de transferencia de materia entre ambas fases para el período de formación y ascenso de la gota. Para estudiar la aireación de suero residual proveniente de la fabricación de quesos se construyó un tanque provisto de un agitador con regulador de velocidad. La concentración de oxígeno disuelto en el líquido se midió empleando un equipo portátil con sensor descartable, los datos experimentales permitieron hallar la velocidad de disolución del gas a través del tiempo (Eckenfelder, 1991). 
La transferencia de materia desde sólidos a fluidos se llevó a cabo también en un tanque agitado (Miller, 1971) disolviendo esferas de naftaleno en soluciones acuosas de etanol. Se determinó la variación de la concentración de naftaleno en un fluido tomando periódicamente muestras y analizándolas con un espectrofotómetro. Como en los casos antes descritos, se compararon los valores de las experiencias con las correlaciones propuestas en la literatura. Se efectuaron estudios paramétricos variando la velocidad característica y se analizó el rango de validez de las expresiones aplicadas. Debe observarse que en los Trabajos Prácticos que involucraron Transferencia de Materia, dadas las necesidades que se presentaron (empleo de instrumental para las determinaciones analíticas y aplicación de técnicas volumétricas de análisis) se contó con la colaboración de otros laboratorios de la Facultad de Ingeniería Química especializados en el área.

Finalmente, además de los trabajos agrupados, se cita una experiencia encuadrada en el tema Transferencia de Cantidad de Movimiento, en la cual debido a sus características particulares se usa un equipo perteneciente al Instituto de Tecnología de Alimentos (F.I.Q.-U.N.L.), obviándose la etapa de diseño y construcción del dispositivo. En dicho Trabajo Practico se midió la viscosidad aparente de distintos fluidos no newtonianos y se obtuvieron los parámetros característicos de acuerdo a modelos teóricos que representan su comportamiento (Papanastasiou, 1994). Se utilizó un reómetro Haake Rotovisco con dos geometrías: cilindros concéntricos y cono-plato y se trabajó con fluidos alimenticios (yogurt, caramelo liquido, ketchup, queso fundido, mayonesa, dulce de leche, pulpa de tomate) y con dispersiones de macromoléculas como la goma guar y aislado proteico de soja.

\section{CONCLUSIONES}

El desarrollo del Trabajo Práctico da lugar a que el estudiante aborde situaciones que pueden resolverse según variados enfoques, propiciando de esta manera la estimulación del pensamiento creativo, el análisis crítico, el razonamiento y la iniciativa personal de los estudiantes. También posibilita una relación más intensa con Centros de Documentación, Gabinete de Informática, Institutos de Investigación, Cátedras y Laboratorios, Talleres de Vidrio y Mecánico, y Servicios de Planta Piloto.

Se ha observado gran interés por parte de los estudiantes en participar en actividades de este tipo, motivados por la posibilidad de ver su diseño construido y utilizado. Además se evidenció sentido de responsabilidad debido a que los resultados por ellos obtenidos serán utilizados en los cursos sucesivos, y el logro de los objetivos propuestos sirve al aprendizaje de todos.

El trabajo en grupo favorece la formación en el campo de las relaciones interpersonales y el desarrollo de capacidades de liderazgo, lo cual resulta beneficioso para el futuro Ingeniero considerando que la carencia de estos atributos va en desmedro de su desempeño profesional, aún cuando cuente con las capacidades técnicas requeridas. Aunque la metodología aplicada exige de los docentes mayor capacitación, dedicación, esfuerzo y tiempo, por otro lado resulta una fuente de estímulos y una experiencia gratificante.

La realización del Trabajo Práctico contribuye a un conocimiento más profundo del alumno y por lo tanto se lo puede evaluar mejor, ya que las oportunidades de interacción con ellos van más allá de los horarios de clases. Además brinda buenas oportunidades para el ejercicio de la comunicación lo cual es útil, teniendo en cuenta la dificultad real que representa para los estudiantes de Ingeniería la expresión oral y escrita.

\section{AGRADECIMIENTOS}

Los autores agradecen a la Universidad Nacional del Litoral (Proy. CAI+D 93-94. N 181 y CAI+D 96. № 203) por su contribución al presente trabajo.

\section{REFERENCIAS}

Albizzati, E. y A. Arese; Experiencias innovadoras en la enseñanza y el aprendizaje de los Fundamentos de la Ingeniería Química, Memorias del III Encuentro Interamericano de Educación en Ingeniería y Tecnología, Río de Janeiro, Brasil (1994) 
Camacho Rubio, F., P. González Tello y E Jurado Alameda; Extracción líquido-líquido en columna de gotas, Anales de Química, Serie A: 77, 256-263 (1981)

Concari, S., G. Alzugaray, A. Arese,y R. Pozo; El trabajo de investigación como recurso didáctico en la enseñanza de la Física, Memorias del I Encuentro Internacional de Educación en Física y III Encuentro Nacional de Profesores de Física, Montevideo, Uruguay (1992)

Crosby, E.J.: Experimentos sobre Fenómenos de Transporte en las Operaciones Unitarias de la Industria Química, Hispano Americana S.A., Buenos Aires (1968)

Eckenfelder, W.W., Jr.; Principles of water quality management, Krieger Publishing Company, Florida (1991)

Garg, H.P. y U. Rani; Theoretical and experimental studies on collectors/storage type solar water heater, Solar Energy: 29(6), 467-478 (1982)

Giorgetti, M.F.: Uma experiência na interface. Experimento de laboratório - meio audiovisual para o ensino de mecânica dos fluidos. Seminario Regional Latinoamericano y del Caribe sobre Enseñanza Experimental de la Ingeniería, Santa Fe, Argentina (1985).

Greenkom, R.A. y D. P. Kessler; Transfer Operations, Mc Graw-Hill Book Company, New York (1972).

Incropera F. y D. De Witt; Fundamentals of heat and mass transfer, 3rd. Ed. J. Wiley \& Sons, New York (1990).

Meinardy, M. y otros cuatro autores; Perfiles teóricos y experimentales de la concentración de sal durante la maduración de quesos, $X$ Congreso Regional de Iniciación Científica y Tecnológica en Ingeniería Caixas do Sul, Brasil (1995).

Miller, D.; Scale-up of agitated vessels, Ind. Eng. Chem. Process Des. Develop.: 10(3), 365-375 (1971)

Molyneux, F.; Ejercicios de Laboratorio de Ingeniería Química, Blume, Madrid (1969).

Nérici, I.; Metodología de la enseñanza Kapelusz Mexicana, México (1980)

Papanastasiou, T.; Applied Fluid Mechanics, Prentice Hall, New Jersey (1994)

Ray Bowen, J.: Engineering Education for the 21st. Century, III Encuentro Interamericano de Educación en Ingeniería y Tecnología, Rio de Janeiro, Brasil (1994).

Reid R., J. Prauznits y B. Poling; The properties of gases and Liquids, 4th. Ed. Mc Graw Hill, New York (1987).

Salinas de Sandoval, J., D. Gil Pérez y L.C. de Cudmani; La elaboración de estrategias educativas acordes con un modo científico de tratar las cuestiones. Memorias IX Reunión de Educación en Física (REF IX), 336-349, Salta (1995a).

Salinas de Sandoval. J., D. Gil Pérez y L. C de Cudmani; ¿Cómo adecuar las estrategias educativas a los requerimientos de modelos de aprendizaje basados en psicologías constructivistas? Memorias IX Reunión de Educación en Física (REF IX), 350-362, Salta (1995b)

Scarrah, W.; The design of a Unit Operations Laboratory based on Piagetian concepts, The Modern undergraduate laboratory: innovative techniques, AIChE Symposium Series. American Institute of Chemical Engineering: 75(183), 26-28 (1979). 